\title{
Shifts in attentional scope modulate event-related potentials evoked by reward
}

\author{
Ajay Nadig ${ }^{1}$ (I) $\cdot$ Nicholas J. Kelley ${ }^{1} \cdot$ Narun Pornpattananangkul ${ }^{1,2,3} \cdot$ James E. Glazer $^{1} \cdot$ Robin Nusslock $^{1}$
}

Published online: 11 March 2019

(C) The Psychonomic Society, Inc. 2019

\begin{abstract}
Emotions broaden or narrow the scope of attention in order to facilitate adaptive responses in threatening and rewarding contexts. In the current study, rather than asking how emotions influence attentional scope, we considered the possibility that the relationship between attentional breadth and emotion is bidirectional by asking whether shifts in attentional scope alter emotional processes using an event-related potential $(E R P)$ paradigm. Participants $(N=30)$ completed a modified version of a Monetary Incentive Delay (MID) task, wherein their attention was either narrowed or broadened as they attempted to win rewards. Behaviorally, narrowing attention improved task performance in the form of reduced errors and increased monetary winnings. During cue processing, narrowing (compared to broadening) attention reduced the Cue-P3 (irrespective of cue type). During feedback processing, narrowing (compared to broadening) attention reduced the Feedback-P3 to monetary wins and increased the Feedback-P2 and the Feedback-P3 to monetary non-wins. Results highlight complexity and bidirectionality in the relationship between attentional scope and affective processes.
\end{abstract}

Keywords Positive affect $\cdot$ Reward $\cdot$ Approach motivation $\cdot$ Attentional scope

\section{Introduction}

Different behavioral contexts place distinct demands on the attentional system, which can flexibly parse a scene in many ways. For example, within a complex scene, one can attend to small details, broad features, or anything in between. Attentional scope is the position of focus along this continuum of perceptual detail. In the classic example of a forest scene, focusing on the most global features (i.e., the forest) requires the broadest attentional scope, whereas focusing on the most local features (i.e., the tree) requires the narrowest attentional

Ajay Nadig and Nicholas J. Kelley shared first-authorship.

Ajay Nadig

ajay.g.nadig@gmail.com

Nicholas J. Kelley

nicholasjkelley@gmail.com

1 Department of Psychology, Northwestern University, Swift Hall, 2029 Sheridan Road, Evanston, IL 60208, USA

2 National Institute of Health and University of Otago, Dunedin, New Zealand

3 Department of Psychology, University of Otago, Dunedin 9016, New Zealand scope. Past research indicates that affective states modulate attentional scope to facilitate adaptive responses to affectively charged stimuli (Easterbrook, 1959; Friedman \& Förster, 2010). The present study extends this work by examining whether shifts in attentional scope reciprocally modulate affective states, with a focus on reward processing.

\section{The broaden-and-build theory}

The relationship between attentional scope and emotion has been frequently studied through the lens of the broaden-andbuild theory (Fredrickson, 1998; Fredrickson, 2013), which suggests that negative emotions narrow attentional scope whereas positive emotions broaden attentional scope. Proponents find that negative mood states and traits are associated with a local bias in attentional scope, whereas positive mood states and traits are associated with a global bias in attentional scope (Basso, Schefft, Ris, \& Dember, 1996; Gasper \& Clore, 2002; Johnson, Waugh, \& Fredrickson, 2010; Rowe, Hirsh, \& Anderson, 2007). However, inconsistent findings (Finucane \& Whiteman, 2007; Huntsinger, Clore, \& Bar-Anan, 2010; Huntsinger, 2012) highlight the need for continued research on the relationship between attentional scope and emotional processes. 


\section{The motivational intensity theory of attentional scope}

One possible reason for the limited generalizability of the broaden-and-build theory is that proponents of the theory conducted studies which confounded valence and motivational intensity by considering only high-intensity negative affect (e.g., fear) and low-intensity positive affect (e.g., happiness or amusement). Both high-intensity positive affect (e.g., desire) and low-intensity negative affect (e.g., sadness) were neglected in prior studies. Inspired by this confound, the motivational intensity theory of attentional scope proposes emotions high in motivational intensity (e.g., fear, anger, desire) narrow attention whereas emotions low in motivational intensity (e.g., sadness, amusement) broaden attention (Domachowska et al., 2016; Gable \& Harmon-Jones, 2008; Gable \& Harmon-Jones, 2010; Threadgill \& Gable, 2018; for review, see Harmon-Jones, Gable, \& Price, 2013). Thus, the influence of emotion on attentional scope may arise not only from an emotion's valence but also from its motivational intensity.

\section{Rewards and spatial attention}

Finally, a separate literature has characterized how reward influences spatial attention. This body of work raises the important point that reward may shape spatial attention independent of affective state. These studies have demonstrated that irrelevant distractor stimuli associated with a reward can involuntarily capture attention in subsequent unrelated tasks (Anderson, Laurent, \& Yantis, 2011) and that such rewarddriven attentional capture can persist long after initial reward-learning (Anderson \& Yantis, 2013). The learning of such reward-attention associations may or may not be accompanied by shifts in affective state; these associations continue to exert influence on attention when the stimulus is no longer an object of task-relevant appetitive behavior. While this work demonstrates a relationship between reward and attention, it does not speak to attentional scope (i.e., global vs. local focus) per se. Nonetheless, we take these studies as evidence that (1) reward-processing is a powerful modulator of attentional processes and (2) generalization of results from the present reward paradigm to emotion in general should be cautious.

\section{Reciprocal influences of attentional scope on affective states}

Critically, all of the aforementioned studies have investigated how reward processing/affective states shape attentional processes. A relatively smaller body of work has studied whether attentional processes reciprocally influence affective state. To our knowledge, only two studies have assessed this question. Gable and Harmon-Jones reported that narrowing versus broadening attention with a "Navon-letters" stimulus enhanced the N1 event-related potential (ERP) component to motivationally intense positive (Gable \& Harmon-Jones, 2011) and motivationally intense negative images (Gable \& Harmon-Jones, 2012). Thus, narrowing attention may enhance the influence of affective stimuli on early attentional processes.

The studies by Gable and Harmon-Jones $(2011,2012)$ have two key limitations. First, they assessed affective processes via neural responses to passively viewed images of dessert foods. Passively obtained rewards do not evoke as strong a response as rewards, which stem from an individual's own effort. Support for this viewpoint comes from research on effort justification (Aronson \& Mills, 1959), which finds that individuals who had to work hard to be initiated into a group (versus those who did not) liked that group more. More recently, others have extended this work to consumer behavior and find that participants report liking products more if they put them together compared with identical products put together by others (i.e., the IKEA effect, see Norton, Mochon, \& Ariely, 2012). More recently, Dohle, Rall, and Siegrist (2014) found that participants reported liking foods (i.e., milkshakes) more that were self-prepared compared with foods prepared by an experimenter. Collectively, this research suggests that effort and effortful tasks increase liking relative to passive tasks. This research suggests that although passively viewed images can evoke affective processes, they are less ideal for evoking motivationally intense states (compared with active behavioral tasks), because they do not require effort from the participant. Accordingly, passive viewing paradigms may be less optimal for maximizing variation in ERPs evoked by affective stimuli like rewards (Martin \& Potts, 2011).

A second related limitation concerns the number of ERPs that can be assessed in passive imaging paradigms. Because these paradigms do not require responses from participants, researchers are not able to study ERPs evoked by responsecontingent stimuli, such as feedback. Given these limitations, these studies highlight the need to examine the effects of attentional scope one emotional processes in an active paradigm. Reward tasks in particular may be well suited to assess the effects of attentional scope on ERPs, because (1) reward tasks often require behavioral responses and (2) can include response-contingent stimuli, allowing for a broader ERP assessment.

\section{The monetary incentive delay task}

To address these issues, we incorporated an attentional scope manipulation into a frequently used response paradigm for eliciting motivated neural processes: the monetary incentivedelay task (MID; Knutson, Westdorp, Kaiser, \& Hommer, 2000). In the standard MID task, trials begin with a reward (or no-reward) cue, then participants perform a button press in 
response to a target object (e.g., a white square), and then immediately after the response, feedback appears on the screen indicating whether they responded fast enough. In our Navon-MID task (Fig. 1), cue and feedback portions are identical to the standard MID. The target object is replaced, however, with a hierarchical Navon-letter image (a large letter made of smaller letters, where those letters are either an $\mathrm{S}, \mathrm{H}$ or O; Fig. 1). Instead of making a simple button press to the target object, the participant must press one of two buttons $(\mathrm{H}$ or S) to identify either the local (the small letter) or global (the large letter) feature of the image (Fig. 1). Past research has demonstrated that attentional scope can successfully be manipulated by presenting blocks of global and local trials (Brand \& Johnson, 2014; Flevaris, Bentin, \& Robertson, 2011; Lachmann, Schmitt, Braet, \& van Leeuwen, 2014; Pletzer, Petasis, \& Cahill, 2014). We incorporated a similar blocking procedure into our Navon-MID task. In local trial blocks, the participant wins if they press the button corresponding to the local letter quickly. In the global trial blocks, the participant wins if they press the button corresponding to the global letter fast enough. This Navon-MID task, as opposed to a passive-viewing paradigm, (1) facilitates participants engaging cognitive and behavioral resources to attain rewards, and (2) leverages well-studied reward cue- and feedback-locked ERPs to investigate how attentional scope modulates reward processing.

\section{Event-related potentials in the context of reward}

We examined the influence of attentional scope on several ERPs across the full temporal range of reward processing. Reward processing involves a wide range of neural processes that subserve the anticipation and consumption of rewards (Berridge \& Robinson, 2003; Knutson, Fong, Adams, Varner, \& Hommer, 2001; Salamone \& Correa, 2012; Breiter, Aharon, Kahneman, Dale, \& Shizgal, 2001; McClure, Berns, \& Montague, 2003). A multitude of ERPs occur at these different stages of reward processing and capture distinct, often orthogonal, aspects of reward processing (Glazer, Kelley, Pornpattanangkul Mittal \&; Nusslock, 2018).
Anticipatory ERPs During the anticipation phase, we analyzed the Cue-N1, Cue-P2, and Cue-P3 ERPs, locked to the cue indicating presence or absence of a monetary incentive. The Cue-N1 is a negative-going frontocentral component peaking approximately 50 to $150 \mathrm{~ms}$ after the presentation of a cue signaling an opportunity to earn a reward (or not). The N1 is enhanced for motivationally relevant stimuli, such as emotional pictures (Foti, Hajcak, \& Dien, 2009; Keil et al., 2001; although see Codispoti, Ferrari, and Bradley (2007) for evidence that emotional pictures does not modulate the N1). The Cue-P2 is a positive-going centrally maximal component, which peaks approximately $200-250 \mathrm{~ms}$ after the cue onset and is elevated in the presence of cues signaling potential rewards in a gambling task (Yu \& Zhou, 2006), a visual discrimination task (Schevernels, Krebs, Santens, Woldorff, \& Boehler, 2014) and in the MID task (Doñamayor, Schoenfeld, \& Münte, 2012). The Cue-P3 is a positivegoing centroparietal component peaking 300 to $500 \mathrm{~ms}$ after cue presentation. The Cue-P3 indexes contextupdating processes in working memory as the cue is categorized (Polich, 2007; Bonala \& Jansen, 2012) and is enhanced by the presence of a motivating incentive (Goldstein et al., 2006; Hughes, Mathan, \& Yeung, 2013; Pornpattananangkul \& Nusslock, 2015).

Feedback ERPs During the feedback processing phase, we analyzed the Feedback-P2, RewP, and Feedback-P3. The Feedback-P2 is a positive-going, centrally maximal component that peaks approximately $180-230 \mathrm{~ms}$ after feedback and was recently found to be enhanced by rewarding versus nonrewarding feedback in a MID task (Doñamayor et al., 2012). Although the $\mathrm{P} 2$ evoked by feedback in active incentive tasks is relatively understudied, the broader family of $\mathrm{P} 2$ components has been shown to be enhanced by arousing versus nonarousing stimuli (Carretié, Hinojosa, Albert, \& Mercado, 2006) and active versus passive tasks (Potts, 2004). This suggests that the P2 generally facilitates selective attention towards task-relevant stimuli (Luck \& Hillyard, 1994; Potts, Martin, Burton, \& Montague, 2006).

\begin{tabular}{|c|c|c|c|c|c|c|c|c|c|c|c|c|c|c|c|c|}
\hline \multirow[t]{2}{*}{ A Trials } & 16 & 16 & 16 & 16 & 16 & 16 & 16 & 16 & 16 & 16 & 16 & 16 & 16 & 16 & 16 & 16 \\
\hline & $\mathrm{L}$ & $\mathrm{G}$ & $\mathrm{L}$ & $\mathrm{G}$ & $\mathrm{L}$ & $\mathrm{G}$ & $\mathrm{L}$ & G & L & G & $\mathrm{L}$ & $\mathrm{G}$ & $L$ & $\mathrm{G}$ & $\mathrm{L}$ & $\mathrm{G}$ \\
\hline
\end{tabular}

B
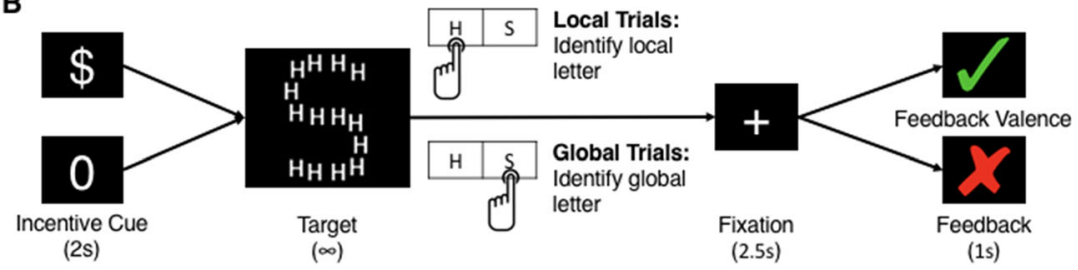

Fig. 1. Schematic of the Navon-MID task. Note: Incentive cues were randomly distributed within each block. Feedback was based on performance and due to the adaptive algorithm, participants won approximately $50 \%$ of the time 
The RewP is a positive-going frontocentral component peaking around $250 \mathrm{~ms}$ after feedback presentation (Hajcak, Moser, Holroyd, \& Simons, 2006; Gehring \& Willoughby, 2002; Yeung \& Sanfey, 2004). The RewP is enhanced by positive feedback valence (e.g., wins or correct feedback) and higher relative feedback value (Sambrook \& Goslin, 2015), and likely, at least in part, reflects reward prediction error (Walsh \& Anderson, 2012).

The Feedback-P3 is a positive-going centroparietal component peaking 250 to $450 \mathrm{~ms}$ after feedback presentation. The Feedback-P3 reflects categorization and motivational intensity of feedback (Sato et al., 2005; Bellebaum, Polezzi, \& Daum, 2010; San Martín, 2012). Importantly, although the Cue-P3 and Feedback-P3 share comparable temporospatial properties, they are largely uncorrelated and likely reflect distinct neural processes of anticipatory preparation for the upcoming trial (Cue-P3) and outcome-based updating of taskmodels to optimize future behavior (Feedback-P3) (Novak \& Foti, 2015: Pornpattananangkul \& Nusslock, 2015).

\section{The current study}

In the current study, we sought to provide the first test of the effect of attentional scope on neural processes during reward anticipation and feedback processing using ERPs. Recall that the broaden-and-build theory predicts that positive emotions are associated with a global bias in attentional scope. Accordingly, based on the broaden-and-build theory, a global attentional scope may broadly enhance reward-ERPs during both anticipate and feedback. The motivational intensity theory predicts that emotions high in motivational intensity are associated with a local bias in attentional scope, whereas emotions low in motivational intensity are associated with a global bias in attentional scope. The motivational intensity theory views reward anticipation as high in motivational intensity and feedback processing as low in motivational intensity. Accordingly, based on the motivational intensity theory, a local attentional scope may enhance ERPs during reward anticipation (namely the N1 as in Gable \& Harmon-Jones, 2011, 2012), whereas a global attentional scope would enhance ERPs during feedback processing.

\section{Method}

\section{Participants}

Thirty-three undergraduate students (16 females) reported individually to a laboratory study described as an investigation of how people process words, sentences, and pictures. Participants ages ranged from 18 to $22(M=19.00$, standard deviation $[S D]=0.87$ ). All participants were healthy (i.e., no history of neurological disorder or use of psychotropic medications) and were strongly right-handed $(<18$ on Chapman Handedness Questionnaire; see Chapman \& Chapman, 1987). Data from three participants were excluded due to EEG equipment error $(n=1)$, excessive movement artifacts ( $>75$ percent of trials, $n=1$ ), and ERP values that were more than three standard deviations away from the mean $(n$ $=1$ ), resulting in a final sample of 30 participants ( 14 females). Participants received credit toward a course requirement for their participation and had the opportunity to win money based on task performance (see below). This study was approved by the Northwestern University Institutional Review Board, and informed consent was obtained for each participant. Data, scripts, and materials are available on the Open Science Framework: https://osf.io/ez4b6.

Power analysis and sample size determination A priori sample size determination is difficult for repeated measures designs with more than one repeated measures factor because of difficulties estimating error variances used in power calculations (Potvin \& Schutz, 2000). As a result, we used Gable and Harmon-Jones $(2011,2012)$ to guide our sample size determination. In a between-subjects study, Gable and Harmon-Jones (2011) recruited approximately 35 participants per cell and observed a moderate effect size of attentional scope on the N1 ERP. In a similar experiment on the N1, Gable and Harmon-Jones (2012) recruited 29 participants for their within-subjects emotion manipulation. We aimed to recruit a sample size between the sample sizes of these two past studies. Rather than using a between-subjects or mixed-model design as in these two studies, we opted for a withinsubjects design, which affords us greater power to detect an effect (Lakens, 2016). Based on this, our data collection goal was to sample a minimum of 29 participants and terminate data collection at the end of the academic year or when the final sample reached 40 participants. Our final sample of 30 participants with fully useable data was consistent with our goal and consistent with the sample sizes used in past research.

\section{Procedure}

After consent, the experimenter introduced the study and applied the electroencephalography (EEG) equipment. Participants then completed the Navon-MID task while EEG data were recorded.

Navon-MID task The Navon-MID Task (Fig. 1) is a modified version of the monetary-incentive delay task. In the standard MID task (Knutson et al., 2000), trials begin with a reward (or no-reward) cue and participants then perform a button press in response to a target object (e.g., a white square). Immediately after the button press, feedback appears on the screen. In our Navon-MID task, cue and feedback portions remain the same 
as the prototypical MID. The only change is that the target object is a Navon letter rather than a white square (see Fig. 1b for a schematic representation), and participants must identify the local or global letter rather than simply pressing a button.

Each trial begins with either a money (\$) or no-money (0) cue, which appears for 2 seconds. Participants were able to win $\$ 0.20$ on money cued trials and $\$ 0.00$ on no-money cued trials. The purpose of this money versus no-money manipulation was to assess how the effects of attentional scope on reward processing vary across trials of differing motivational intensity (i.e., money trials: more motivationally intense; nomoney trials: less motivationally intense). After the cue, a Navon-letter appeared. Navon letters are large letters made up of closely spaced smaller letters (an $H$ made up of $S$ s; these figures were composed from every combination of $\mathrm{H}, \mathrm{S}$, and O). Participants are asked to indicate "as quickly as possible" whether the picture contains the letter $S$ or the letter $H$, by pressing one button for $S$ and another button for $H$. The global target is the larger letter, whereas the local target is the smaller letter. On local trials, participants were asked to identify the local target. On global trials, participants were asked to identify the global target. Participants completed alternating blocks for local and global trials (Fig. 1a).

The Navon letter stayed on the screen until the participant responded. After pressing, the participant then saw a fixation cross for 2.5 seconds, followed by presentation of feedback for 1 second. Wins were contingent upon both accuracy and speed. Accuracy was based on correctly identifying the local or global target. Speed was based on an adaptive algorithm, which controls for accuracy at approximately $50 \%$. On the first trial of the Navon-MID, correct responses were those in which participants reaction time was less than their median reaction time on a prior nonincentive version of the NavonMID task. This nonincentive version of the Navon-MID task included 64 trials in total composed of four alternating 16-trial blocks of local and global trials. This nonincentive task was designed solely to establish participant's median reaction time for use in the main task. This median reaction time was used as the time limit for the first trial of the main task. On subsequent trials, the time-window was shortened by $10 \mathrm{~ms}$ if the ratio of positive feedback exceeded 0.55 , lengthened if the ratio of positive feedback was below 0.45 , and maintained if the ratio of positive feedback was between these values. Although previous studies using an EEG version of the MID typically use a 66\%-win ratio (Broyd et al., 2012; Novak \& Foti, 2015, Study 1) we used a method that enforced a near-50\% win ratio to control for feedback frequency effects on outcome-related ERPs (Angus et al., 2017; Novak \& Foti, 2015, Study 2).

For money trials, wins were identified by a green checkmark paired with " $+20 ф$ "; non-wins were identified by a red $\mathrm{X}$ paired with " $+00 \notin$." For no-money trials, wins were identified by a green checkmark paired with " $+00 \notin$ "; non-wins were identified by a red X paired with "+ 00 ф."
Participants completed 16 blocks of 16 trials ( 256 total trials). Attentional scope (local vs. global target) was the blocking variable. All participants began with a block of local trials. In each block, half of the trials were money-cued and half were not money-cued. At the beginning of each trial, participants were reminded of the scope of the current block. Money and nomoney cued trials were randomly distributed within each block. After the cue, all trials in local blocks asked participants to identify local targets before receiving feedback. Likewise, after the cue, all trials in global blocks asked participants to identify global targets before receiving feedback. In summary, this task contains 256 trials in a 2 (Attentional Scope: local vs. global) $\times 2$ (Incentive: money vs. no money) within-subjects design (Fig. 1).

Electrophysiological recording We recorded 64-channel continuous EEG using a NeuroScan system while participants completed the Navon-MID task within an electromagnetically shielded booth. Data were sampled at $500 \mathrm{~Hz}$ (DC to $100 \mathrm{~Hz}$ online). In addition to the 64 scalp channels, two horizontal and two vertical electro-oculogram channels were recorded via facial electrodes for artifact rejection purposes. Data were referenced online to the left mastoid channel and then rereferenced offline to the linked mastoid channels. We ensured that impedances at each scalp and mastoid channel were below $5 \mathrm{k} \Omega$ and that impedances at each facial channel was below $10 \mathrm{k} \Omega$. Movement related artifacts were removed manually. Eyeblinks and saccades were removed using independent components analysis algorithms implemented in EEGLAB software (Delorme \& Makeig, 2004).

\section{Event-related potential analysis}

Data processing was completed using EEGLAB software (Delorme \& Maekig, 2004). After artifact rejection, data were bandpass filtered $(0.1-30 \mathrm{~Hz})$ and digitized at $250 \mathrm{~Hz}$. Data for each participant were epoched using two events: presentation of cue, and presentation of feedback. For both cue and feedback, we epoched the time series from $200 \mathrm{~ms}$ before to $800 \mathrm{~ms}$ after the event and performed baseline correction using the time window from 200 before stimulus presentation to time of stimulus presentation. Artifacts were rejected using three complementary strategies: moving window threshold (removing epochs $>100 \mu \mathrm{V}$ ), epochs with greater than $50 \mu \mathrm{V}$ between two adjacent samples, and epochs with flatlined channels. On average, $8.58 \%$ of trials were removed for the cue epoch (SD = 4.93), and $9.74 \%$ of trials were removed for each participant in the feedback epoch $(\mathrm{SD}=4.91)$. Within each bin, trials were averaged to give mean waveforms for each participant. For the cue epoch, on average 58.5 trials were included per bin (Local/ Global x Money/No Money) per participant ( $\mathrm{SD}=3.2$ trials). For the feedback epoch, on average 28.9 trials were included per bin (Local/Global x Money/No Money x Correct/Incorrect) per participant $(\mathrm{SD}=1.6$ trials). 
Temporo-spatial principal components analysis Temporospatial PCA was conducted separately on cue and feedback data using the ERP PCA Toolkit (Dien, 2010b) following recommended guidelines for PCA analysis on ERP components (Dien, 2010a; Dien, Beal, \& Berg, 2005; Dien, Khoe, \& Mangun, 2007). We used PCA because most of the ERPs of interest occur within a short time window $(\sim 200 \mathrm{~ms}$ to $\sim 500 \mathrm{~ms}$ post-stimulus). Although these components have varying scalp distributions, the poor spatial resolution of EEG means that these components may "bleed" into each other even in grand average waveforms, confounding measurement (Luck, 2014). This has proven especially problematic in the case of ERPs evoked by rewards, such as RewP and $\mathrm{P} 3$, which have shown inconsistencies in the literature, likely due to overlap between neighboring components elicited close together in time (Sambrook \& Goslin, 2015). To address this issue, temporo-spatial PCA examines the distribution of EEG data the post-cue time window, the post-feedback time window, and across different scalp channels, to extract temporospatially defined factors that explain a large proportion of data variance. The ERP PCA Toolkit has been successfully used in many experiments to extract and quantify ERPs (Carlson, Foti, Mujica-Parodi, Harmon-Jones, \& Hajcak, 2011; Foti et al., 2011; Weinberg, Riesel, \& Proudfit, 2014).

We conducted temporal PCA using ProMax rotation and extracted the appropriate number of factors based on the resulting Scree plot (Cattell, 1966). Eleven temporal factors were extracted from cue data, and ten temporal factors were extracted from feedback data. Following the temporal PCA, we performed spatial PCA using infomax rotation and again extracted the appropriate number of factors based on the resulting Scree plot. We removed factors less than $\pm 1 \mu \mathrm{V}$ in amplitude. Together, the remaining factors accounted for $89.8 \%$ of the total variance in the grand average ERP waveform for the cue epoch and $89.9 \%$ of the total variance in the grand average ERP waveform for the feedback epoch. After this, we extracted four factors for both cue and feedback data (Fig. 2). These extracted factors displayed latencies and scalp topographies consistent with common cue- and feedback-evoked ERPs implicated in reward processing. Thus, we extracted the Cue-P2, FeedbackP2, Cue-P3, Feedback-P3, and RewP (Glazer, Kelley, Pornpattanangkul, Mittal, \& Nusslock, 2018), as well as the Cue-N1, which most closely aligns to the N1 component studied by Gable and Harmon-Jones $(2011,2012)$. Together, these factors accounted for $32.9 \%$ of the total variance in the grand average ERP waveform for the cue epoch and $33.5 \%$ of the total variance in the grand average ERP waveform for the feedback epoch. All PCAs implemented covariance matrix and Kaiser Normalization (as recommended by Dien, 2010a). Finally, factor waveforms were reconstructed through conversion to microvolts (Fig. 2). Factor scores were quantified by taking the mean activity $\pm 50 \mathrm{~ms}$ around their peak at the electrode site where peak voltage is maximal.

\section{Results}

\section{Analysis plan}

Task winnings were evaluated in a one-way within-subjects ANOVA to comparing participant's earnings in local money-cued trials and global money-cued trials. Errors, reactions times, and anticipatory ERPs were evaluated in a series of 2 (Attentional Scope: local vs. global) $\times 2$ (Incentive: money vs. no money) within-subjects ANOVAs. Finally, feedback-related ERPs were evaluated in a series of 2 (Attentional Scope: local vs. global) $\times 2$ (Incentive: money vs. no money) $\times 2$ (Feedback: Correct vs. Incorrect) within-subjects ANOVAs. We employed Fisher's protected $t$ tests (Cohen, Cohen, West, \& Aiken, 1983) to minimize familywise error rate which requires a significant omnibus ANOVA $F$ test in to proceed to pairwise comparisons.

\section{Behavior}

Task winnings To assess the consequences of attentional scope on task monetary winnings, we performed a within-subjects ANOVA to compare participant's earnings in local money-cued trials and global money-cued trials (Fig. 3a). Participants earned significantly less money on global money-cued $(M=\$ 6.95$, standard error $[S E]=0.26$, 95\% confidence interval [CI] [\$6.41, \$7.49]) compared with local money-cued $(M=\$ 8.76, S E=0.22,95 \%$ CI $[\$ 8.30, \$ 9.21])$ trials, $F(1,29)=28.77, p<0.001$, partial $\eta^{2}=0.50$.

Errors To assess the behavioral consequences of attentional scope and incentive in more detail, we analyzed number of errors in a 2 (Attentional Scope: local vs. global) $\times 2$ (Incentive: money vs. no money) within-subjects ANOVA (Fig. 3b). Participants made more errors on global trials $(M$ $=11.55, S E=0.90,95 \%$ CI $[9.71,13.39])$ versus local trials $(M=4.82, S E=0.53,95 \%$ CI $[3.74,5.89]), F(1,29)=56.16$, $p<0.001$, partial $\eta^{2}=0.70$. Participants also made more errors on money trials $(M=8.90, S E=0.71,95 \%$ CI $[7.44,10.36])$ versus no-money trials $(M=7.47, S E=0.52,95 \%$ CI [6.40, $8.53]), F(1,29)=10.52, p=0.003$, partial $\eta^{2}=0.27$. These main effects were qualified by an attentional scope $\times$ incentive interaction, $F(1,29)=8.93, p=0.006$, partial $\eta^{2}=0.24$. Simple main effects revealed that on global trials, participants made more errors on money trials $(M=13.03, S E=1.11,95 \%$ CI $[10.76,15.31])$ compared with no-money trials $(M=10.07$, $S E=0.83,95 \%$ CI $[8.37,11.76]), F(1,29)=14.59, p<0.001$, partial $\eta^{2}=0.34$. On local trials, participants made a nonsignificantly different amount of errors on money trials $(M=$ $4.77, S E=0.60,95 \%$ CI $[3.54,5.99])$ compared with no- 


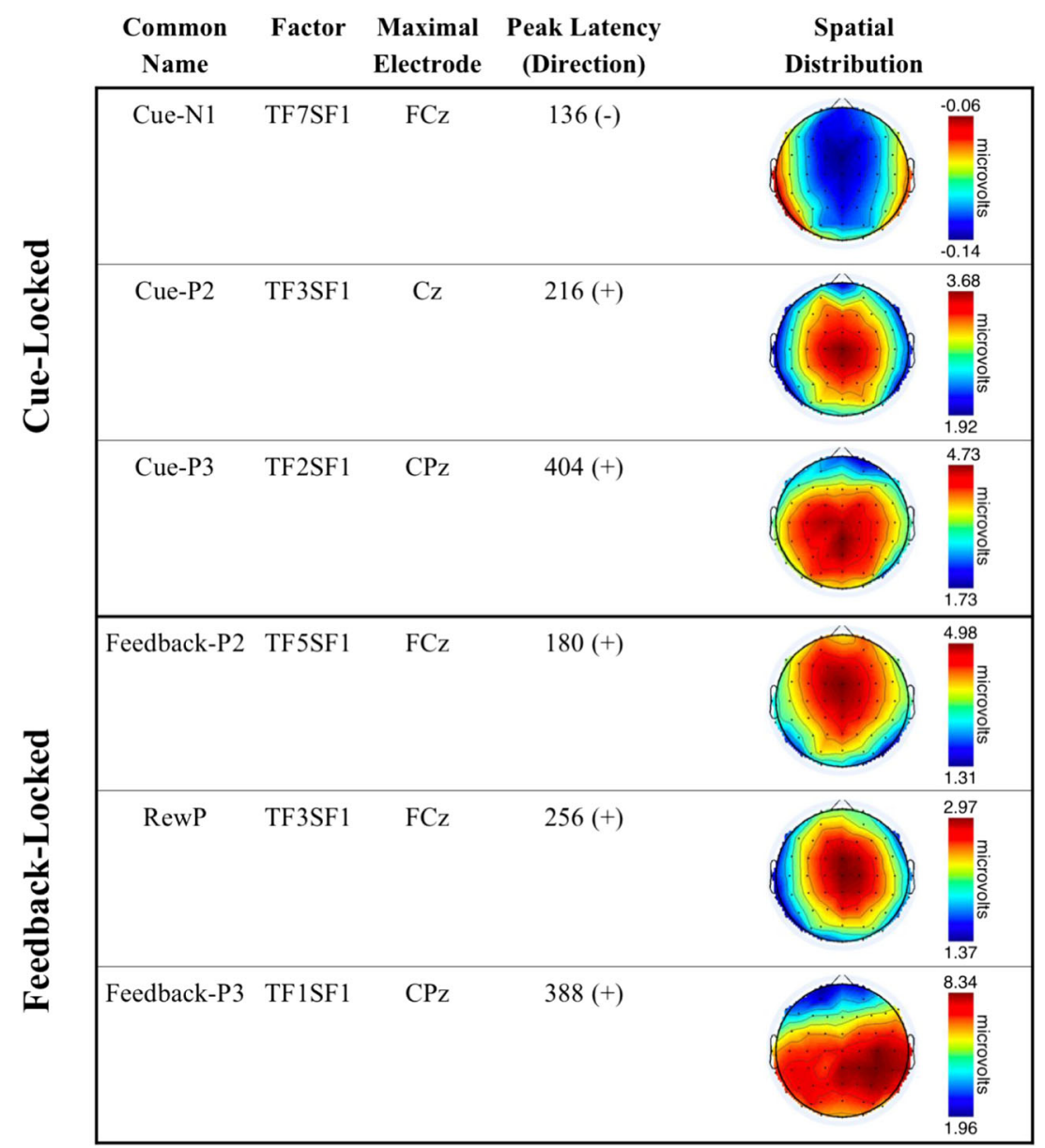

Fig. 2. PCA components. After visual inspection, PCA-derived factors temporospatially resembling ERPs of interest were extracted and analyzed. Topographic maps were generated by averaging at each electrode from $50 \mathrm{~ms}$ before to $50 \mathrm{~ms}$ after peak latency
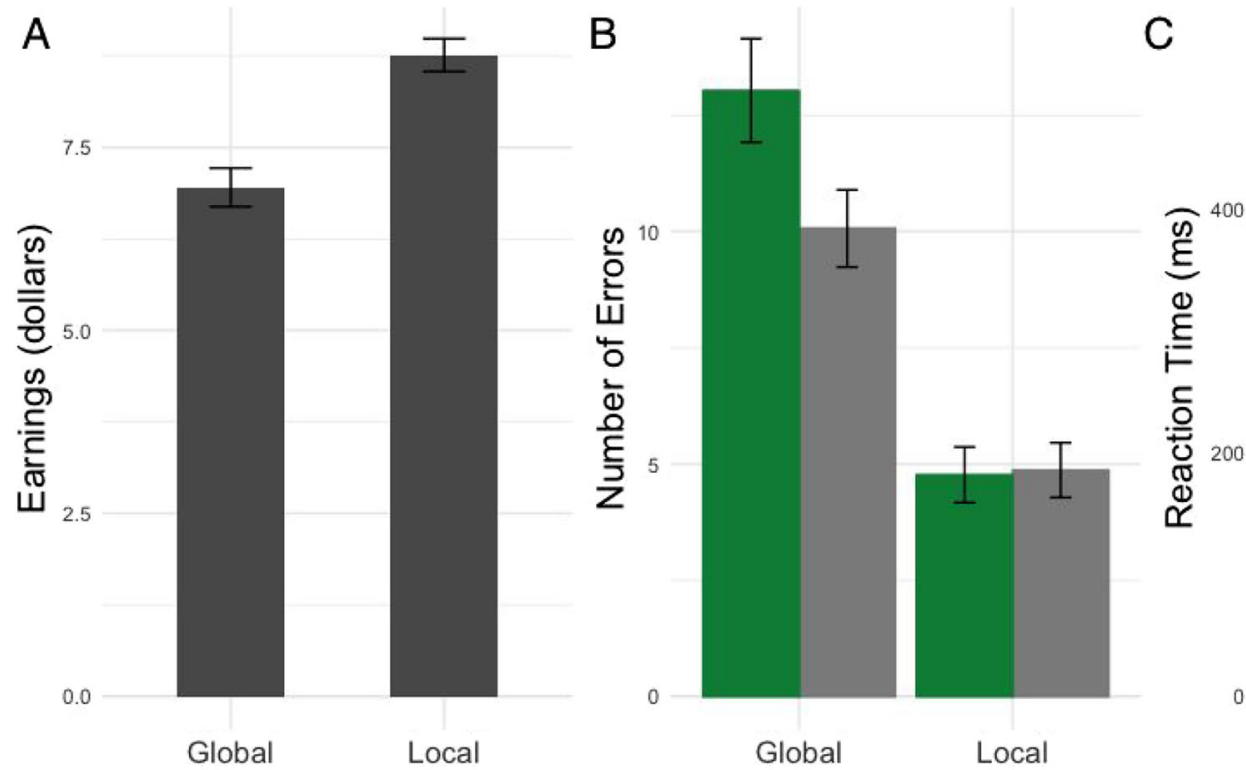

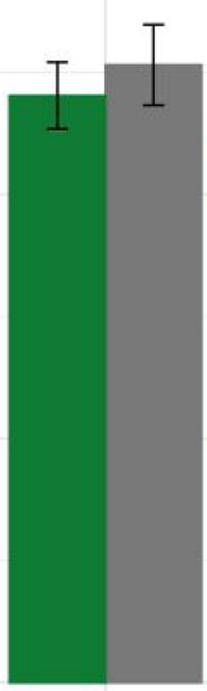

Global

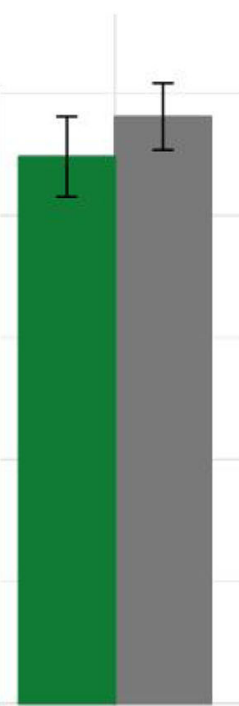

Local

Fig. 3. Behavioral results from the attentional scope monetary incentive delay task. (a) Monetary earnings as a function of trial type. (b) Errors as a

Reaction time as a function of incentive (Money $=$ Green; No Money $=$ Grey) and Scope. Note: Error bars reflect \pm 1 standard error of the mean 
money trials $(M=4.87, S E=0.59,95 \%$ CI $[3.66,6.08]), F(1$, 29) $=0.03, p=0.86$, partial $\eta^{2}=0.001 .^{1}$

Reaction time To ensure that the adaptive algorithm effectively controlled reaction time differences between local and global trials, we analyzed average reaction times in a 2 (Attentional Scope: local vs. global) $\times 2$ (Incentive: money vs. no money) within-subjects ANOVA (Fig. 3c). Reaction times were non-significantly different on global trials $(M=465.25 \mathrm{~ms}, S E=20.89 \mathrm{~ms})$ compared with local trials $(M=464.62 \mathrm{~ms}, S E=25.34 \mathrm{~ms}, 95 \%$ CI [412.79, 516.45]), $F(1,29)=0.002, p=0.97$, partial $\eta^{2}<0.001$. Reaction times were not significantly faster on money trials $(M=436.27 \mathrm{~ms}, S E=23.13 \mathrm{~ms}, 95 \%$ CI [388.97, 483.57]) compared with no-money trials $(M=493.60 \mathrm{~ms}$, $S E=28.48 \mathrm{~ms}, 95 \%$ CI $[435.34,551.85]), F(1,29)=4.12$, $p=0.052$, partial $\eta^{2}=0.12$. There was no attentional scope $\times$ incentive interaction, $F(1,29)=2.60, p=0.12$, partial $\eta^{2}$ $=0.08$. These results suggest that the adaptive task design was generally effective at controlling reaction times.

Interim summary: behavioral results In summary, narrowing attention enhanced performance on the Navon-MID as reflected in fewer errors and greater earnings.

\section{Reward cue evaluation ERPs}

Cue-N1 A 2 (Attentional Scope: local vs. global) $\times 2$ (Incentive: money vs. no money) within-subjects ANOVA was conducted to assess the effects of attentional scope and incentive on the Cue-N1 (Fig. 4b). The magnitude of the CueN1 was non-significantly different on local trials $(M=-0.58$, $S E=0.16,95 \%$ CI $[-0.912,-0.249])$ compared with global trials $(M=-0.79, S E=0.18,95 \%$ CI $[-0.1 .15,-0.45]), F(1$, 29) $=1.93, p=0.18$, partial $\eta^{2}=0.06$. The magnitude of the Cue-N1 was nonsignificantly different on money $(M=-0.61$, $S E=0.20,95 \%$ CI $[-1.02,-0.21])$ compared with no money $(M=-0.76, S E=0.15,95 \% \mathrm{CI}[-1.15,-0.43])$ trials, $F(1,29)$ $=0.61, p=0.44$, partial $\eta^{2}=0.02$. There was a nonsignificant attentional scope $\times$ incentive interaction, $F(1,29)=0.98, p=$ 0.33 , partial $\eta^{2}=0.03$.

\footnotetext{
${ }_{1}^{1}$ Differences in error rates may be due in part to task difficulty. As a result, we conducted all ERP analyses with and without errors entered as a covariate. Errors did not change our results and because of this we did not report analyses with covariates in the manuscript. On an exploratory basis we also examined bivariate correlations among the 36 ERP estimates included in the main analyses ( 3 Cue ERPS in 4 conditions each, 3 Feedback ERPs in 8 conditions each) and the number of errors for each condition. Only $2 / 144$ of the resulting correlations were significant. First, the number of local/no-money errors was significantly correlated with the FB-P2 on global-correct-money trials, $r=$ $-.40, p=.03$. Second, the number of global/no-money errors was significantly correlated with the FB-P2 on global-correct-no money trials, $r=.40, p=.03$. This suggests that error rates appear to have negligible effects on ERPs in the current study.
}

Cue-P2 A 2 (Attentional Scope: local vs. global) $\times 2$ (Incentive: money vs. no money) within-subjects ANOVA was conducted to assess the effects of attentional scope and incentive on the Cue-P2 (Fig. 4c). The Cue-P2 did not differ in magnitude on local trials $(M=2.73, S E=0.40,95 \%$ CI $[1.90,3.56])$ compared with global trials $(M=2.40, S E=0.43,95 \%$ CI $[1.52$, 3.27]), $F(1,29)=3.34, p=0.08$, partial $\eta^{2}=0.10$. The Cue-P2 was larger on money trials $(M=3.56, S E=0.48,95 \%$ CI $[2.58$, 4.54]) compared with no-money trials $(M=1.56, S E=0.35$, $95 \%$ CI $[0.84,2.28]), F(1,29)=74.64, p<0.001$, partial $\eta^{2}=$ 0.72 . The attentional scope $\times$ incentive interaction was not significant, $F(1,29)=3.85, p=0.06$, partial $\eta^{2}=0.12$.

Cue-P3 A 2 (Attentional Scope: local vs. global) $\times 2$ (Incentive: money vs. no money) within-subjects ANOVA was conducted to assess the effects of attentional scope and incentives on the Cue-P3 (Fig. 4d). The magnitude of the CueP3 was smaller on local trials $(M=4.48, S E=0.69,95 \% \mathrm{CI}$ $[3.08,5.89])$ compared with global trials $(M=5.15, S E=0.70$, $95 \%$ CI $[3.72,6.59), F(1,29)=5.03, p=0.03$, partial $\eta^{2}=$ 0.15 . The magnitude of the Cue-P3 was larger on money trials $(M=5.42, S E=0.78,95 \%$ CI $[3.81,7.03])$ compared with nomoney trials $(M=4.21, S E=0.64,95 \% \mathrm{CI}[2.90,5.52]), F(1$, $29)=6.52, p=0.02$, partial $\eta^{2}=0.18$. There was a nonsignificant attentional scope $\times$ incentive interaction, $F$ (1, 29) $=0.12, p=0.73$, partial $\eta^{2}=0.004$.

Interim summary: reward anticipation ERPs In summary, we observed no effects of attentional scope on the Cue-N1 or CueP2 and found that broadening attention enhances the Cue-P3.

\section{Reward outcome ERPs}

Feedback-P2 A 2 (Attentional Scope: local vs. global) $\times 2$ (Incentive: money vs. no money) $\times 2$ (Feedback: Correct vs. Incorrect) within-subjects ANOVA was conducted to assess the effects of attentional scope, incentive, and feedback on the Feedback-P2 (Fig. 5b). Feedback-P2 amplitudes were non-significantly different on local trials $(M=3.44, S E=$ $0.42,95 \%$ CI $[2.58,4.30])$ compared with global trials $(M=$ $3.21, S E=0.44,95 \%$ CI $[2.32,4.10]), F(1,29)=1.68, p=$ 0.21 , partial $\eta^{2}=0.06$. Feedback-P2 amplitudes were larger on money trials $(M=3.78, S E=0.47,95 \%$ CI $[2.83,4.73])$ compared with no-money trials $(M=2.87, S E=0.39,95 \% \mathrm{CI}$ $[2.08,3.66)$ trials, $F(1,29)=28.53, p<0.001$, partial $\eta^{2}=$ 0.50 . Feedback-P2 amplitudes were also larger on correct trials $(M=4.06, S E=0.45,95 \%$ CI $[3.13,4.99])$ compared with incorrect trials $(M=2.59, S E=0.42 .95 \%$ CI $[1.73,3.45]), F$ $(1,29)=31.09, p<0.001$, partial $\eta^{2}=0.52$.

Next, we observed a significant Attentional Scope $\times$ Incentive interaction, $F(1,29)=4.58, p=0.04$, partial $\eta^{2}=$ 0.14 . Simple main effects revealed that on money trials, the magnitude of the Feedback-P2 was larger on local trials $(M=$ 


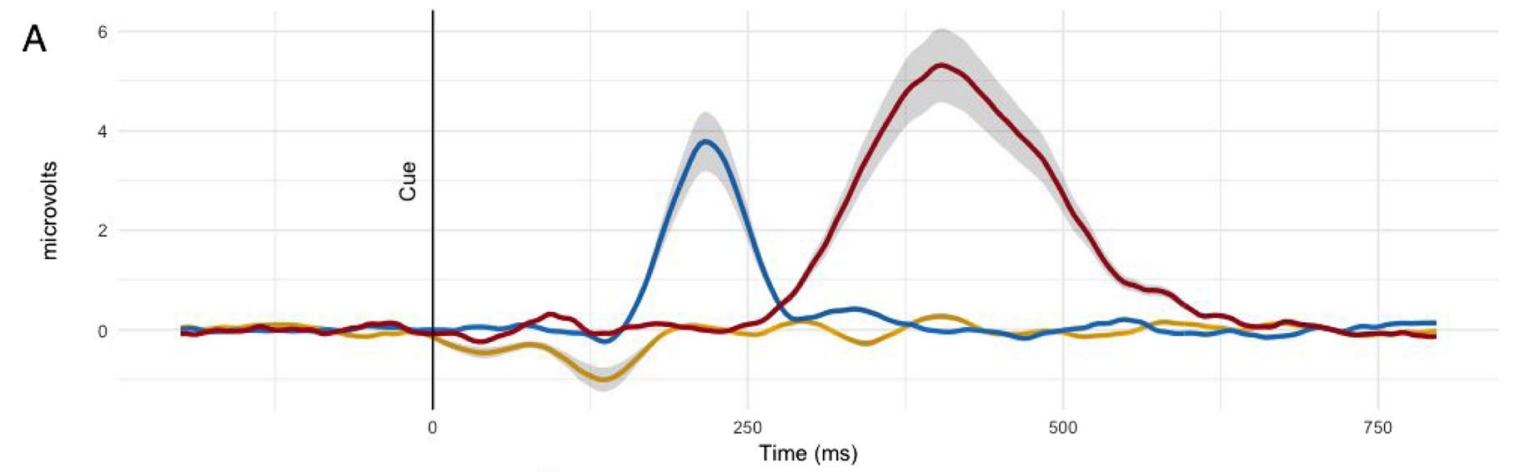

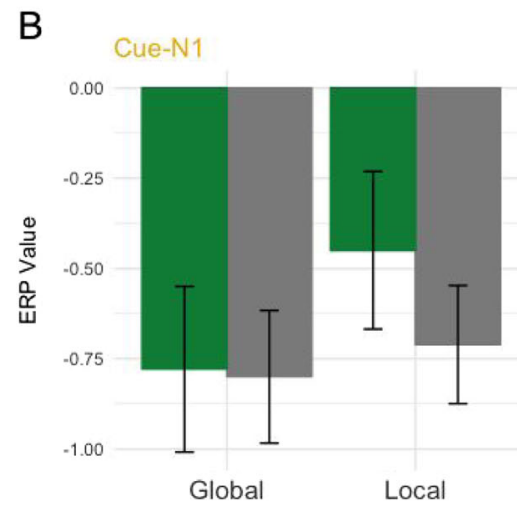

Fig. 4. PCA factor scores for cue ERPs. (a) Cue-locked time series of isolated PCA components (Cue-N1: Orange; Cue-P2: Blue; Cue-P3: Red). Bar graphs depicting the Cue-N1 (b), Cue-P2 (c), and Cue-P3 (d)

4.10, $S E=.46,95 \%$ CI $[3.17,5.03])$ compared with global trials $(M=3.47, S E=0.50,95 \%$ CI $[2.44,4.50]), F(1,29)=$ 6.93, $p=0.01$, partial $\eta^{2}=0.19$. In no-money trials, there was no difference in Feedback-P2 amplitudes between local trials $(M=2.79, S E=0.42,95 \%$ CI $[1.94,3.64])$ and global trials $(M$ $=2.30, S E=0.43,95 \%$ CI $[1.42,3.18]), F(1,29)=0.40, p=$ 0.54 , partial $\eta^{2}=0.01$.

Second, we observed a significant Attentional Scope $\times$ Feedback interaction, $F(1,29)=4.48, p=0.04$, partial $\eta^{2}=$ 0.13 . For correct feedback, the magnitude of the Feedback-P2 was nonsignificantly different on local trials $(M=4.00, S E=$ $0.45,95 \%$ CI $[3.08,4.91])$ compared with global trials $(M=$ 4.13, $S E=0.49,95 \%$ CI $[3.12,5.14]), F(1,29)=0.29, p=$ 0.59 , partial $\eta^{2}=0.01$. For incorrect feedback, the magnitude of the Feedback-P2 was larger on local trials $(M=2.88, S E=$ $0.45,95 \%$ CI $[1.96,3.80])$ compared with global trials $(M=$ 2.23, $S E=0.43,95 \%$ CI $[1.42,3.18]), F(1,29)=5.72, p=$ 0.02 , partial $\eta^{2}=0.17$.

Finally, we did not observe a significant Incentive $\times$ Feedback interaction, $F(1,29)=3.07, p=0.09$, partial $\eta^{2}=$ 0.10 or a three-way interaction, $F(1,29)=0.89, p=0.35$, partial $\eta^{2}=0.03$.

Reward positivity A 2 (Attentional Scope: local vs. global) $\times 2$ (Incentive: money vs. no money) $\times 2$ (Feedback: Correct vs. Incorrect) within-subjects ANOVA was conducted to assess the effects of attentional scope, incentive, and feedback on
D Cue-P3

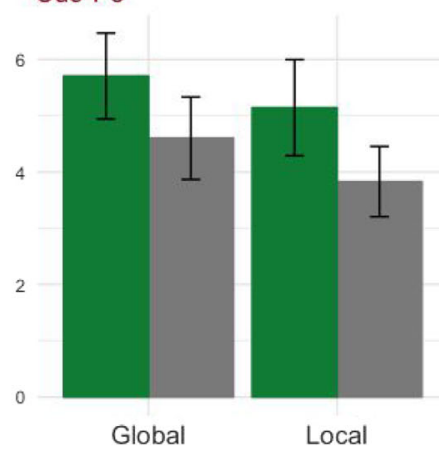

are shown as a function of attentional scope and incentive (Money Trials: Green; No Money Trials: Grey). Note: Error bars reflect \pm 1 standard error of the mean

the RewP (Fig. 5c). The RewP was larger for correct $(M=$ $3.68, S E=0.55,95 \%$ CI $[2.56,4.80])$ compared with incorrect feedback $(M=1.02, S E=0.46,95 \%$ CI $[0.08,1.96]), F(1,29)$ $=85.46, p<0.001$, partial $\eta^{2}=0.75$. The RewP did not differ significantly for local trials $(M=2.19, S E=0.47,95 \% \mathrm{CI}$ $[1.23,3.15])$ compared with global trials $(M=2.51, S E=$ $0.51,95 \%$ CI $[1.46,3.56]), F(1,29)=3.39, p=0.08$, partial $\eta^{2}=0.11$. The RewP did not differ significantly between money trials $(M=2.59, S E=0.53,95 \%$ CI $[1.52,3.67])$ and no-money trials $(M=2.11, S E=0.47,95 \%$ CI $[1.14,3.07]), F$ $(1,29)=3.89, p=0.06$, partial $\eta^{2}=0.12$.

There was no significant Incentive $\times$ Feedback interaction $\left[F(1,29)=3.63, p=0.07\right.$, partial $\left.\eta^{2}=0.11\right]$, Scope $\times$ Feedback $\left[F(1,29)=1.19, p=0.29\right.$, partial $\left.\eta^{2}=0.04\right]$ or Scope $\times$ Incentive $\left[F(1,29)=1.26, p=0.27\right.$, partial $\left.\eta^{2}=0.04\right]$ interactions. Finally, the three-way interaction also was not significant, $F(1,29)=1.50, p=0.23$, partial $\eta^{2}=0.05$.

Feedback-P3 A 2 (Attentional Scope: local vs. global) $\times 2$ (Incentive: money vs. no money) $\times 2$ (Feedback: Correct vs. Incorrect) within-subjects ANOVA was conducted to assess the effects of attentional scope, incentive, and feedback on the P3 (Fig. 5d). The magnitude of the Feedback-P3 was nonsignificantly different on local trials $(M=8.45, S E=0.68$, $95 \%$ CI $[7.17,9.74])$ compared with global trials $(M=8.28$, $S E=0.64,95 \%$ CI $[6.98,9.58]), F(1,29)=0.49, p=0.49$, partial $\eta^{2}=0.02$. The magnitude of the Feedback-P3 was 


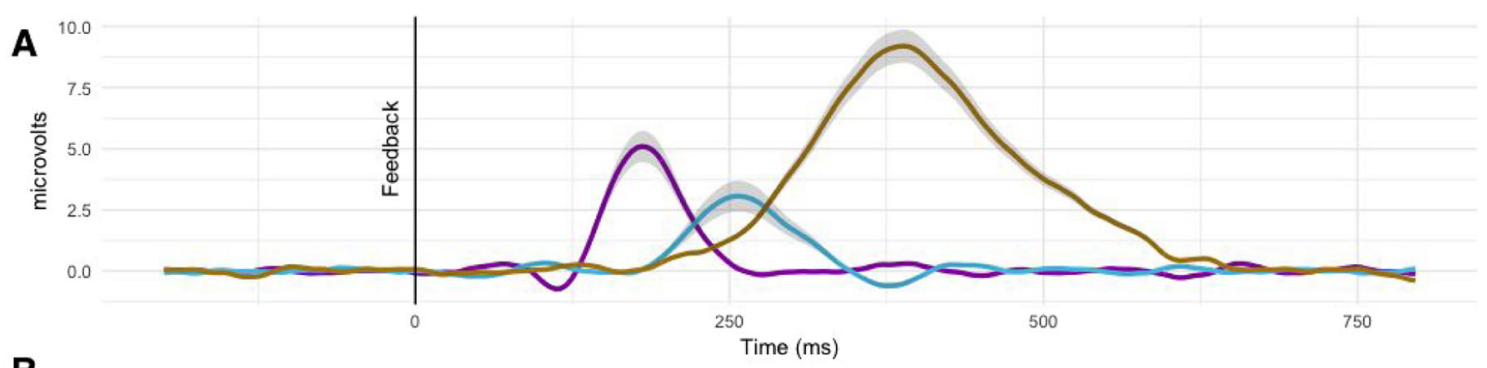

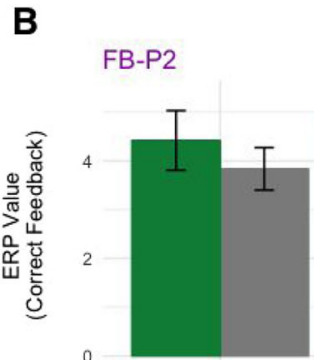

Global

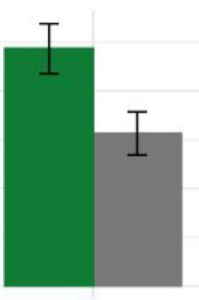

Local

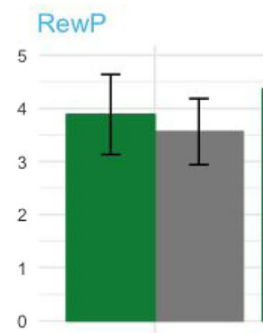

Global

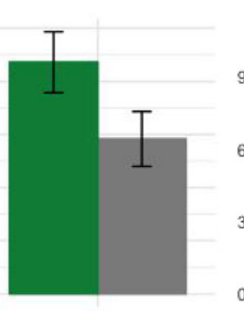

Local

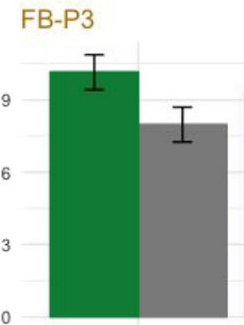

Global

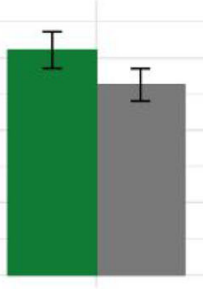

Local

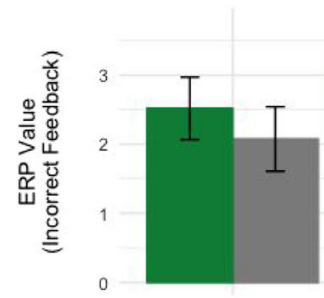

Global

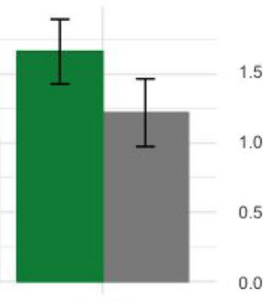

Local

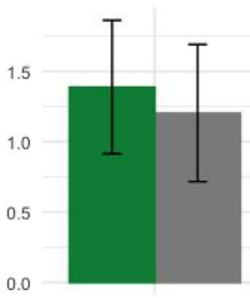

Global

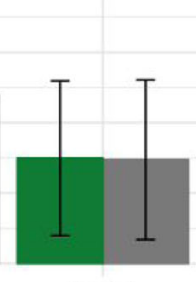

Local

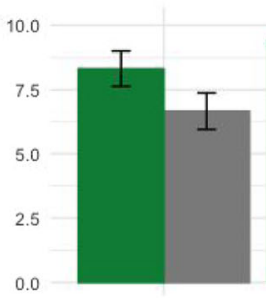

Global

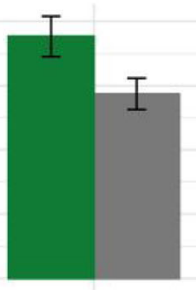

Local
Fig. 5. PCA factor scores for feedback ERPs. (a) Feedback-locked time series of isolated PCA components (Feedback-P2: Purple; RewP: Teal; Feedback-P3: Gold). Bar graphs depicting (b) the Feedback-P2, (c) the
RewP, and (d) Feedback-P3 are shown as a function of attentional scope, incentive, and Feedback valence (Money Trials: Green; No Money Trials: Grey). Note: FB = Feedback, Error bars reflect \pm 1 standard error of the mean larger on money trials $(M=9.30, S E=0.68,95 \%$ CI [7.90, $10.70])$ compared with no-money trials $(M=7.43, S E=0.60$, $95 \%$ CI $[6.20,8.66]), F(1,29)=26.62, p<0.001$, partial $\eta^{2}=$ 0.51 . The magnitude of the Feedback-P3 was larger on correct trials $(M=8.83, S E=0.65,95 \%$ CI $[7.51,10.15])$ compared with incorrect trials $(M=7.90, S E=0.63,95 \%$ CI $[6.60$, 9.20]), $F(1,29)=8.17, p=0.008$, partial $\eta^{2}=0.22$.

Both the Attentional Scope $\times$ Incentive $[F(1,29)=$ 0.03, $p=0.87$, partial $\left.\eta^{2}=0.001\right]$ and the Feedback $\times$ Incentive $\left[F(1,29)=0.10, p=0.75\right.$, partial $\left.\eta^{2}=0.003\right]$ interactions were nonsignificant. There was a significant Attentional Scope $\times$ Feedback interaction, $F(1,29)=$ 5.61, $p=0.03$, partial $\eta^{2}=0.16$.

Critically, this two-way interaction above was qualified by a significant three-way interaction, $F(1,29)=5.45, p=$ 0.03 , partial $\eta^{2}=0.16$. To unpack the three-way interaction, we examined the attentional scope $\times$ feedback simple interaction separately for money trials and no-money trials. For money trials, there was a significant attentional scope $\times$ feedback interaction, $F(1,29)=11.96, p=0.002$, partial $\eta^{2}=0.29$. We further decomposed this simple interaction by examining the simple main effects scope separately for correct and incorrect trials. On correct trials, the magnitude of the Feedback-P3 was larger on global trials $(M=10.14$, $S E=0.72,95 \%$ CI $[8.66,11.62])$ compared with local trials $(M=9.32, S E=0.75,95 \%$ CI $[7.78,10.86]), F(1$, $29)=5.44, p=0.03$. On incorrect trials, we observed an opposite pattern whereby the magnitude of the FeedbackP3 was smaller on global trials $(M=8.32, S E=0.68,95 \%$ CI $[6.92,9.72])$ compared with local trials $(M=9.42, S E=$ $0.78,95 \%$ CI $[7.82,11.01]), F(1,29)=5.44, p=0.02$. There was no attentional scope $\times$ feedback interaction for no money trials, $F(1,29)=0.90, p=0.35$, and as a result we did not decompose this interaction any further.

Interim summary: reward outcome ERPs The attentional scope manipulation did not modulate the RewP, which was sensitive only to feedback and incentive. We observed effects of attentional scope on the Feedback-P2 and Feedback-P3 whereby (1) narrowing attention enhanced the Feedback-P2 for money versus no money trials, and incorrect versus correct feedback, and (2) broadening attention enhanced the Feedback-P3 for correct feedback and narrowing attention enhanced the Feedback-P3 for incorrect feedback, where both of these effects were present only for money trials. 


\section{Discussion}

The present study was designed to characterize how attentional scope affects behavioral and neural systems engaged by a performance-based reward task. We extend previous research reporting that affective states modulate attentional scope by demonstrating that the reverse is also true, namely that attentional scope modulates affective states. Specifically, we report that attentional scope affects both reward-related behavior and electrocortical responses underlying cue and feedback reward processing. We further demonstrate that the influence of attentional scope on reward-related ERPs is not uniform but rather dependent on several factors, including the temporal window, the type of feedback (i.e., correct vs. incorrect), and the presence (vs. absence) of an incentive. We report that (1) broadening attention enhances the Cue-P3, (2) narrowing attention enhances the Feedback-P2 to negative feedback, and (3) when an incentive is present, narrowing attention enhances Feedback-P3 to negative feedback. whereas broadening attention enhances Feedback-P3 to positive feedback. These findings highlight the influence of attentional breadth on reward processing.

\section{Narrowing attention enhances motivated behavior}

We observed significant effects of attentional scope on participant earnings and errors. Participants earned significantly more in local versus global trials, which was driven by a reduction in errors during local versus global trials. We also observed increased errors during money versus no-money trials but only during global trials. These results suggest that narrowing attention enhances task performance and that motivational factors, such as incentive, differentially affect task performance, depending on the scope of attention.

In the current study, we observed that participants made more errors on global compared with local trials. We acknowledge that our design is not well-suited to resolve conclusively whether these behavioral effects arise from the attentional scope manipulation or a difference in task difficulty between local and global conditions. Navon (1977) originally observed that participants demonstrate a global bias at baseline. It also is known that effort is inherently aversive (Inzlicht, Shenhav, \& Olivola, 2018). Given this, it seems unlikely that individuals baseline mode of processing (global processing) would be more difficult than local processing. Regardless of the source of these behavioral differences, the difference in error rates may complicate interpretation of the ERP results. However, this concern is mitigated in our experiment, because inclusion of errors as a covariate did not change our results. Nonetheless, future studies that incorporate attentional scope manipulations into active incentive tasks should attempt to reproduce these behavioral results.

\section{Effects of attentional scope manipulation on cue-evoked ERPs}

We report that broadening versus narrowing attention enhances reward processing during the anticipatory period, as indexed by the Cue-P3. This finding was unexpected and at odds with our behavioral results where narrowed attention lead to greater task winnings. This finding suggests that broadening attention may modulate the cue evaluation process indexed by the Cue-P3 in a manner similar to motivating incentive, which also enhanced the Cue-P3 (although there was no observed interaction between incentive and attentional scope). Furthermore, we did not find statistically significant differences in the Cue-P2 following our attentional scope manipulation. This result, when contrasted with our Cue-P3 finding, highlights the utility of analyzing ERPs across the processing stream, as these nearby components show different profiles of attentional scope modulation. Last, we did not observe an effect of the attentional scope manipulation on the Cue-N1. This result suggests that N1 effects observed by Gable and Harmon-Jones (2011) may hold in passive viewing tasks but not active tasks designed to evoke motivated behavior.

\section{Effects of attentional scope manipulation on feedback-evoked ERPs}

We observed a pattern of effects whereby manipulating attentional scope differentially affected early attentional processes and motivational salience during the reward-outcome phase in a manner that was dependent on both incentive and feedback type. The presence of a monetary incentive enhanced early attentional allocation to feedback, as indexed by the Feedback-P2, after attention was narrowed, but not after attention was broadened. Furthermore, incorrect versus correct feedback enhanced the Feedback-P2 after attention was narrowed but not after attention was broadened. During subsequent reward processing, we find that narrowing versus broadening attention impacts feedback motivational salience, as indexed by the Feedback-P3, in a valence dependent manner. Specifically, narrowing attention enhances the FeedbackP3 to incorrect feedback, whereas broadening attention enhances the Feedback-P3 to correct feedback.

The Feedback-P3 finding above is the first demonstration of a bidirectional relationship where narrowing and broadening attention produce opposite profiles of feedback-dependent effects, suggesting a dynamic relationship between attentional scope and valence processing. Furthermore, these results are only found in the presence of a monetary incentive, suggesting that such valence effects are contingent on motivational processes. Building on the Feedback-P2 finding, this suggests that the effects of valence and motivational intensity on reward-outcome are not entirely dissociated, but rather interactive. 


\section{Limitations and future directions}

One limitation of the current study was the omission of cues which signaled potential monetary non-win (in addition to reward and no-reward cued trials) and punishing feedback (in addition to rewarding and non-rewarding feedback). Using a task design such as this in conjunction with an attentional scope manipulation would allow us to more completely characterize the effects of narrowing and broadening attention on reward processing. Previous research has reported that reward and non-win related ERPs are context dependent and sensitive to their relative value within a task (Holroyd, Larsen, \& Cohen, 2004). Based on this logic, a task which included reward, neutral, and non-win trials may produce comparable findings to the present study for the reward/nonwin contrast while dampening effects observed in reward/ neutral contrast.

Furthermore, the present study did not vary reward magnitude to minimize task length and complexity. Future research should map these effects across different reward magnitudes to assess whether the effects observed with a small reward in this study generalize to larger rewards. Individual trials of this task gave participants very small rewards, which are not likely to individually generate strong emotional responses and do not map well onto the intense stimuli relevant to clinical interests (e.g., drugs, gambling, etc.) This may explain the small effect sizes observed for some attentional scope effects; these effects may be larger when larger rewards are used.

An additional limitation was the relatively low number of trials per condition in the feedback epoch. Whereas cue-ERPs are examined as a function of Incentives and scope feedbackERPs include an additional factor: correct versus incorrect feedback. As a result, feedback ERPs include half the number of trials compared to cue ERPs. With regard to the feedbackERPs we assessed, 20 trials are sufficient to obtain both a reliable RewP (Marco-Pallares, Cucurell, Münte, Strien, \& Rodriguez-Fornells, 2011) and P300 (Cohen \& Polich, 1997). In the current study, every participant's average number of trials per trial type was greater than 20. Across 240 bins of trials ( 30 participants $\times 8$ trial types) only 18 bins (approximately $7 \%$ ) have a trial count below 20 . Of those 18 bins below 20 trials, all but 1 are above 15 . This suggests that although trial counts are substantially reduced in the feedback epoch, the trial counts were still large enough to produce reliably feedback ERPs.

Finally, research on the relationship between reward and spatial attention (Anderson et al., 2011; Anderson \& Yantis, 2013) suggests that associations between reward-processing and attention are not necessarily accompanied by shifts in emotion. Based on this reasoning, it is unclear whether relationships between reward processing and attentional scope in the current study were accompanied by shifts in emotion. Future studies should examine whether or not our scope manipulations shifts causes shifts in emotional states and whether these subsequent shifts modulate reward processing.

\section{Conclusions}

These results demonstrate the surprising capacity of simple attentional manipulations to impact downstream affective neural processes and behaviors. Given that direct manipulation of the brain systems that drive motivated behavior is invasive and impractical, yet critical to clinical interests, manipulations that indirectly alter affect are attractive due to their ease and low cost. We suggest that manipulation of attention scope may, with further development and refinement, serve as a potent, highly practical modulator of affect. Theoretically, we add to the literature more detailed evidence that the relationship between attentional scope and emotional phenomena is bidirectional.

Acknowledgments Preparation of this manuscript was supported by National Institute of Health (NIH) grant T32 NS047987 to NJK and R01-MH100117-01A1 from the National Institute of Mental Health to RN.

\section{References}

Anderson, B. A., Laurent, P. A., \& Yantis, S. (2011). Value-driven attentional capture. Proceedings of the National Academy of Sciences, 108(25), 10367-10371.

Anderson, B. A., \& Yantis, S. (2013). Persistence of value-driven attentional capture. Journal of Experimental Psychology: Human Perception and Performance, 39(1), 6.

Angus, D. J., Latham, A. J., Harmon-Jones, E., Deliano, M., Balleine, B., \& Braddon-Mitchell, D. (2017). Electrocortical components of anticipation and consumption in a monetary incentive delay task. Psychophysiology, 54(11), 1686-1705.

Aronson, E., \& Mills, J. (1959). The effect of severity of initiation on liking for a group. The Journal of Abnormal and Social Psychology, 59(2), 177

Basso, M. R., Schefft, B. K., Ris, M. D., \& Dember, W. N. (1996). Mood and global-local visual processing. Journal of the International Neuropsychological Society: JINS, 2(3), 249-255.

Bellebaum, C., Polezzi, D., \& Daum, I. (2010). It is less than you expected: The feedback-related negativity reflects violations of reward magnitude expectations. Neuropsychologia, 48(11), 3343-3350.

Berridge, K. C., \& Robinson, T. E. (2003). Parsing reward. Trends in Neurosciences, 26(9), 507-513.

Bonala, B. K., \& Jansen, B. H. (2012). A computational model for generation of the P300 evoked potential component. Journal of Integrative Neuroscience, 11(3), 277-294.

Brand, J., \& Johnson, A. P. (2014). Attention to local and global levels of hierarchical Navon figures affects rapid scene categorization. Frontiers in Psychology, 5, 1274.

Breiter, H. C., Aharon, I., Kahneman, D., Dale, A., \& Shizgal, P. (2001). Functional imaging of neural responses to expectancy and experience of monetary gains and non-wins. Neuron, 30(2), 619-639.

Broyd, S. J., Richards, H. J., Helps, S. K., Chronaki, G., Bamford, S., \& Sonuga-Barke, E. J. S. (2012). An electrophysiological monetary incentive delay (e-MID) task: A way to decompose the different 
components of neural response to positive and negative monetary reinforcement. Journal of Neuroscience Methods, 209(1), 40-49.

Carlson, J. M., Foti, D., Mujica-Parodi, L. R., Harmon-Jones, E., \& Hajcak, G. (2011). Ventral striatal and medial prefrontal BOLD activation is correlated with reward-related electrocortical activity: A combined ERP and fMRI study. NeuroImage, 57(4), 1608-1616.

Carretié, L., Hinojosa, J. A., Albert, J., \& Mercado, F. (2006). Neural response to sustained affective visual stimulation using an indirect task. Experimental Brain Research, 174(4), 630-637.

Cattell, R. B. (1966). The scree test for the number of factors. Multivariate Behavioral Research, 1(2), 245-276.

Chapman, L. J., \& Chapman, J. P. (1987). The measurement of handedness. Brain and Cognition, 6(2), 175-183.

Codispoti, M., Ferrari, V., \& Bradley, M. M. (2007). Repetition and event-related potentials: Distinguishing early and late processes in affective picture perception. Journal of Cognitive Neuroscience, 19(4), 577-586.

Cohen, J., Cohen, P., West, S. G., \& Aiken, L. S. (1983). Applied multiple regression/correlation analysis for the behavioral sciences. New York: Psychology Press. https://doi.org/10.4324/9781410606266.

Cohen, J., \& Polich, J. (1997). On the number of trials needed for P300. International Journal of Psychophysiology, 25(3), 249-255.

Delorme, A., \& Makeig, S. (2004). EEGLAB: An open source toolbox for analysis of single-trial EEG dynamics including independent component analysis. Journal of Neuroscience Methods, 134(1), 921.

Dien, J. (2010a). Evaluating two-step PCA of ERP data with geomin, infomax, oblimin, promax, and varimax rotations. Psychophysiology, 47(1), 170-183.

Dien, J. (2010b). The ERP PCA Toolkit: An open source program for advanced statistical analysis of event-related potential data. Journal of Neuroscience Methods, 187(1), 138-145.

Dien, J., Beal, D. J., \& Berg, P. (2005). Optimizing principal components analysis of event-related potentials: Matrix type, factor loading weighting, extraction, and rotations. Clinical Neurophysiology: Official Journal of the International Federation of Clinical Neurophysiology, 116(8), 1808-1825.

Dien, J., Khoe, W., \& Mangun, G. R. (2007). Evaluation of PCA and ICA of simulated ERPs: Promax vs. Infomax rotations. Human Brain Mapping, 28(8), 742-763.

Dohle, S., Rall, S., \& Siegrist, M. (2014). I cooked it myself: preparing food increases liking and consumption. Food Quality and Preference, 33, 14-16.

Domachowska, I., Heitmann, C., Deutsch, R., Goschke, T., Scherbaum, S., \& Bolte, A. (2016). Approach-motivated positive affect reduces breadth of attention: Registered replication report of Gable and Harmon-Jones (2008). Journal of Experimental Social Psychology, $67,50-56$.

Doñamayor, N., Schoenfeld, M. A., \& Münte, T. F. (2012). Magneto- and electroencephalographic manifestations of reward anticipation and delivery. NeuroImage, 62(1), 17-29.

Easterbrook, J. A. (1959). The effect of emotion on cue utilization and the organization of behavior. Psychological Review, 66(3), 183-201.

Finucane, A. M., \& Whiteman, M. C. (2007). Positive emotions induced by massage do not broaden attention and cognition. The Irish Journal of Psychology, 28(3-4), 139-152.

Flevaris, A. V., Bentin, S., \& Robertson, L. C. (2011). Attention to hierarchical level influences attentional selection of spatial scale. Journal of Experimental Psychology: Human Perception and Performance, 37(1), 12-22.

Foti, D., Hajcak, G., \& Dien, J. (2009). Differentiating neural responses to emotional pictures: Evidence from temporal-spatial PCA. Psychophysiology, 46(3), 521-530.

Fredrickson, B. L. (1998). What good are positive emotions? Review of General Psychology, 2(3), 300-319.
Fredrickson, B. L. (2013). Positive emotions broaden and build. Advances in Experimental Social Psychology, 47(1), 53.

Friedman, R. S., \& Förster, J. (2010). Implicit affective cues and attentional tuning: An integrative review. Psychological Bulletin, 136(5), 875-893.

Gable, P. A., \& Harmon-Jones, E. (2008). Approach-motivated positive affect reduces breadth of attention. Psychological Science, 19(5), 476-482.

Gable, P. A., \& Harmon-Jones, E. (2010). The blues broaden, but the nasty narrows: Attentional consequences of negative affects low and high in motivational intensity. Psychological Science, 21(2), 211-215.

Gable, P. A., \& Harmon-Jones, E. (2011). Attentional states influence early neural responses associated with motivational processes: Local vs. global attentional scope and $\mathrm{N} 1$ amplitude to appetitive stimuli. Biological Psychology, 87(2), 303-305.

Gable, P. A., \& Harmon-Jones, E. (2012). Reducing attentional capture of emotion by broadening attention: Increased global attention reduces early electrophysiological responses to negative stimuli. Biological Psychology, 90(2), 150-153.

Gasper, K., \& Clore, G. L. (2002). Attending to the big picture: Mood and global versus local processing of visual information. Psychological Science, 13(1), 34-40.

Gehring, W. J., \& Willoughby, A. R. (2002). The medial frontal cortex and the rapid processing of monetary gains and non-wins. Science, 295(5563), 2279-2282.

Glazer, J. E., Kelley, N. J., Pornpattanangkul, N., Mittal, V. A., \& Nusslock, R. (2018). Beyond the FRN: Broadening the timecourse of EEG and ERP components implicated in reward processing. International Journal of Psychophysiology, 132, 184-202.

Goldstein, R. Z., Cottone, L. A., Jia, Z., Maloney, T., Volkow, N. D., \& Squires, N. K. (2006). The effect of graded monetary reward on cognitive event-related potentials and behavior in young healthy adults. International Journal of Psychophysiology, 62(2), 272-279.

Hajcak, G., Moser, J. S., Holroyd, C. B., \& Simons, R. F. (2006). The feedback-related negativity reflects the binary evaluation of good versus bad outcomes. Biological Psychology, 71(2), 148-154.

Harmon-Jones, E., Gable, P. A., \& Price, T. F. (2013). Does negative affect always narrow and positive affect always broaden the mind? Considering the influence of motivational intensity on cognitive scope. Current Directions in Psychological Science https://doi.org/ 10.1177/0963721413481353

Holroyd, C. B., Larsen, J. T., \& Cohen, J. D. (2004). Context dependence of the event-related brain potential associated with reward and punishment. Psychophysiology, 41(2), 245-253.

Hughes, G., Mathan, S., \& Yeung, N. (2013). EEG indices of reward motivation and target detectability in a rapid visual detection task. NeuroImage, 64, 590-600.

Huntsinger, J. R. (2012). Does positive affect broaden and negative affect narrow attentional scope? A new answer to an old question. Journal of Experimental Psychology: General, 141(4), 595-600.

Huntsinger, J. R., Clore, G. L., \& Bar-Anan, Y. (2010). Mood and globallocal focus: Priming a local focus reverses the link between mood and global-local processing. Emotion, 10(5), 722.

Inzlicht, M., Shenhav, A., \& Olivola, C. Y. (2018). The effort paradox: Effort is both costly and valued. Trends in Cognitive Sciences, 22(4), 337-349.

Johnson, K. J., Waugh, C. E., \& Fredrickson, B. L. (2010). Smile to see the forest: Facially expressed positive emotions broaden cognition. Cognition \& Emotion, 24(2), 299-321.

Keil, A., Müller, M. M., Gruber, T., Wienbruch, C., Stolarova, M., \& Elbert, T. (2001). Effects of emotional arousal in the cerebral hemispheres: A study of oscillatory brain activity and event-related potentials. Clinical Neurophysiology: Official Journal of the International Federation of Clinical Neurophysiology, 112(11), 2057-2068. 
Knutson, B., Fong, G. W., Adams, C. M., Varner, J. L., \& Hommer, D. (2001). Dissociation of reward anticipation and outcome with eventrelated fMRI. Neuroreport, 12(17), 3683-3687.

Knutson, B., Westdorp, A., Kaiser, E., \& Hommer, D. (2000). FMRI visualization of brain activity during a monetary incentive delay task. NeuroImage, 12(1), 20-27.

Lachmann, T., Schmitt, A., Braet, W., \& van Leeuwen, C. (2014). Letters in the forest: Global precedence effect disappears for letters but not for non-letters under reading-like conditions. Frontiers in Psychology, 5, 705.

Lakens, D. (2016, November 12). Why Within-Subject Designs Require Fewer Participants than Between-Subject Designs [blog post]. Retrieved from http://daniellakens.blogspot.com/2016/11/whywithin-subject-designs-require-less.html

Luck, S. J. (2014). An introduction to the event-related potential technique. MIT Press, Cambridge.

Luck, S. J., \& Hillyard, S. A. (1994). Electrophysiological correlates of feature analysis during visual search. Psychophysiology, 31(3), 291308.

Marco-Pallares, J., Cucurell, D., Münte, T. F., Strien, N., \& RodriguezFornells, A. (2011). On the number of trials needed for a stable feedback-related negativity. Psychophysiology, 48(6), 852-860.

Martin, L. E., \& Potts, G. F. (2011). Medial frontal event-related potentials and reward prediction: Do responses matter? Brain and Cognition, 77(1), 128-134.

McClure, S. M., Berns, G. S., \& Montague, P. R. (2003). Temporal prediction errors in a passive learning task activate human striatum. Neuron, 38(2), 339-346.

Navon, D. (1977). Forest before trees: The precedence of global features in visual perception. Cognitive psychology, 9(3), 353-383.

Norton, M. I., Mochon, D., \& Ariely, D. (2012). The IKEA effect: When labor leads to love. Journal of Consumer Psychology, 22(3), 453460.

Novak, K. D., \& Foti, D. (2015). Teasing apart the anticipatory and consummatory processing of monetary incentives: An eventrelated potential study of reward dynamics. Psychophysiology, 52(11), 1470-1482.

Pletzer, B., Petasis, O., \& Cahill, L. (2014). Switching between forest and trees: Opposite relationship of progesterone and testosterone to global-local processing. Hormones and Behavior, 66(2), $257-266$.

Polich, J. (2007). Updating P300: An integrative theory of P3a and P3b. Clinical Neurophysiology: Official Journal of the International Federation of Clinical Neurophysiology, 118(10), 2128-2148.

Pornpattananangkul, N., \& Nusslock, R. (2015). Motivated to win: Relationship between anticipatory and outcome reward-related neural activity. Brain and Cognition, 100, 21-40.

Potts, G. F. (2004). An ERP index of task relevance evaluation of visual stimuli. Brain and Cognition, 56(1), 5-13.
Potts, G. F., Martin, L. E., Burton, P., \& Montague, P. R. (2006). When things are better or worse than expected: The medial frontal cortex and the allocation of processing resources. Journal of Cognitive Neuroscience, 18(7), 1112-1119.

Potvin, P. J., \& Schutz, R. W. (2000). Statistical power for the two-factor repeated measures ANOVA. Behavior Research Methods, Instruments, \& Computers, 32(2), 347-356.

Rowe, G., Hirsh, J. B., \& Anderson, A. K. (2007). Positive affect increases the breadth of attentional selection. Proceedings of the National Academy of Sciences of the United States of America, 104(1), 383-388.

Salamone, J. D., \& Correa, M. (2012). The mysterious motivational functions of mesolimbic dopamine. Neuron, 76(3), 470-485.

Sambrook, T. D., \& Goslin, J. (2015). A neural reward prediction error revealed by a meta-analysis of ERPs using great grand averages. Psychological Bulletin, 141(1), 213-235.

San Martín, R. (2012). Event-related potential studies of outcome processing and feedback-guided learning. Frontiers in Human Neuroscience, 6, 304.

Sato, A., Yasuda, A., Ohira, H., Miyawaki, K., Nishikawa, M., Kumano, H., \& Kuboki, T. (2005). Effects of value and reward magnitude on feedback negativity and P300. Neuroreport, 16(4), 407-411.

Schevernels, H., Krebs, R. M., Santens, P., Woldorff, M. G., \& Boehler, C. N. (2014). Task preparation processes related to reward prediction precede those related to task-difficulty expectation. NeuroImage, 84 , 639-647.

Threadgill, A. H., \& Gable, P. A. (2018). Negative affect varying in motivational intensity influences scope of memory. Cognition and Emotion. https://doi.org/10.1080/02699931.2018.

Walsh, M. M., \& Anderson, J. R. (2012). Learning from experience: Event-related potential correlates of reward processing, neural adaptation, and behavioral choice. Neuroscience and Biobehavioral Reviews, 36(8), 1870-1884.

Weinberg, A., Riesel, A., \& Proudfit, G. H. (2014). Show me the Money: The impact of actual rewards and non-wins on the feedback negativity. Brain and Cognition, 87, 134-139.

Yeung, N., \& Sanfey, A. G. (2004). Independent coding of reward magnitude and valence in the human brain. The Journal of Neuroscience: The Official Journal of the Society for Neuroscience, 24(28), 62586264.

Yu, R., \& Zhou, X. (2006). Brain responses to outcomes of one's own and other's performance in a gambling task. Neuroreport, 17(16), 17471751.

Publisher's note Springer Nature remains neutral with regard to jurisdictional claims in published maps and institutional affiliations. 